\title{
Multi-level Meta-reasoning with Higher-Order Abstract Syntax
}

\author{
Alberto Momigliano and Simon J. Ambler \\ Department of Mathematics and Computer Science, University of Leicester, \\ Leicester, LE1 7RH, U.K. \\ A.Momigliano@mcs.le.ac.uk, S.Ambler@mcs.le.ac.uk
}

\begin{abstract}
Combining Higher Order Abstract Syntax (HOAS) and (co)induction is well known to be problematic. In previous work [1] we have described the implementation of a tool called Hybrid, within Isabelle HOL, which allows object logics to be represented using HOAS, and reasoned about using tactical theorem proving and principles of (co)induction. Moreover, it is definitional, which guarantees consistency within a classical type theory. In this paper we describe how to use it in a multi-level reasoning fashion, similar in spirit to other meta-logics such $F O \lambda^{\Delta I N}$ and Twelf. By explicitly referencing provability, we solve the problem of reasoning by (co)induction in presence of non-stratifiable hypothetical judgments, which allow very elegant and succinct specifications. We demonstrate the method by formally verifying the correctness of a compiler for (a fragment) of Mini-ML, following [10]. To further exhibit the flexibility of our system, we modify the target language with a notion of non-well-founded closure, inspired by Milner \& Tofte 16] and formally verify via co-induction a subject reduction theorem for this modified language.
\end{abstract}

\section{Introduction}

Higher Order Abstract Syntax (HOAS) is a representation technique, dating back to Church, where binding constructs in an object logic are encoded within the function space provided by a meta-language based on a $\lambda$-calculus. This specification is generic enough that sometimes HOAS has been identified [12] merely with a mechanism to delegate $\alpha$-conversion to the meta-language. While this is important, it is by no means the whole story. As made clear by the LF and $\lambda$ Prolog experience, further benefits come when, for an object logic type $i$, this function space is taken to be $\mathrm{i} \Rightarrow \mathrm{i}$, or a subset of it. Then object-logic substitution can be rendered as meta-level $\beta$-conversion. A meta-language offering this facility is a step up, but there is still room for improvement. Experiments such as the one reported in [17] suggest that the full benefits of HOAS can only be enjoyed when it is paired with hypothetical and parametric judgments. A standard example is the full HOAS encoding of type-inference in which the type environment of a term is captured abstractly without any reference to a list of variable/type pairs. Though this is appealing, such judgments are generally not 
inductive since they may contain negative occurrence of the predicate being defined. This raises the question of how we are going to reason on such encodings, in particular, are there induction and case analysis principles available?

A solution that has emerged in the last five years is that specification and (inductive) meta-reasoning should be dealt with in a single system but at different levels. One such meta-logic is Miller \& McDowell $F O \lambda^{\Delta N}$ [14]; it is is based on higher-order intuitionistic logic augmented with definitional reflection [8] to provide support for case analysis — and induction on natural numbers. Consistency is ensured by cut-elimination. Inside this meta-language they develop a specification logic (SL) which in turn it is used to specify the object-logic (OL) under study. This partition solves the problem of meta-reasoning in the presence of negative occurrences, since hypothetical judgments are now encapsulated within the OL and therefore not required to be inductive. The price to pay is this additional layer where we explicitly reference provability and the necessity therefore of a sort of meta-interpreter (the SL logic) to access it.

Very recently, Felty [6] has suggested that, rather than implementing an interactive theorem prover for $F O \lambda^{\Delta I N}$ from scratch, the latter can be simulated within an existing system (Coq in that case); in particular, definitional reflection is mimicked by the elimination rules of inductive types. Nevertheless, this is not quite enough, as reasoning by inversion crucially depends on simplifying in the presence of constructors. Since some of the higher-order ones may be noninductive, Felty recurs to the assumption of a set of axioms stating the freeness and extensionality properties of constructors in the given signature. Under those conditions the author shows, in the example of the formalization of subject reduction for Mini-ML, how it is possible to replicate, in an well-understood and interactive setting, the style of proofs typical of $F O \lambda^{\Delta N}$; namely the result is proven without "technical" lemmas foreign to the mathematics of the problem.

In previous work [1] we have described the implementation of a higher-order meta-language, called Hybrid, within Isabelle HOL, which provides a form of HOAS for the user to represent object logics. The user level is separated from the infrastructure, in which HOAS is implemented definitionally via a de Bruijn style encoding.

In this paper we adopt most of Felty's architecture, with the notable difference of using Hybrid rather than Coq as the basic meta-language. A graphical depiction of the proposed architecture is in Figure 1 Moreover, we take a further departure in design: we suggest to push at the OL only those judgments which would not be inductive, and to leave the rest at the Isabelle HOL level. We claim that this framework has several advantages:

- The system is more trustworthy: freeness of constructors and more importantly extensionality properties at higher types are not assumed, but proven via the related properties of the infrastructure, see Subsection 3.1 .

- The mixing of meta-level and OL judgments makes proofs more easily mechanizable and allows us to use co-induction which is still unaccounted for in a logic such as $F O \lambda^{\Delta I N}$. 


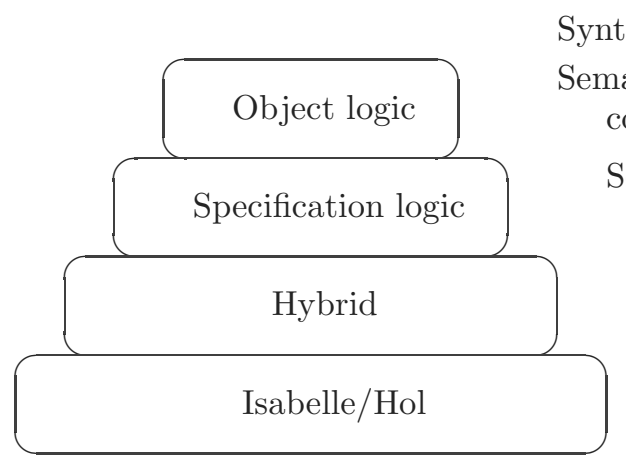

Syntax: lam $x . E x$, fix $x . E x \ldots$

Semantics: typing $\Gamma \vdash E: \tau$, compilation $\eta \vdash F \leftrightarrow e$

Sequent calculus: $\Phi \triangleright_{n} G$, prog clauses, induction on height

Meta-language: "Datatype"

for a $\lambda$-calculus

Tactics/Simplifier

HOL (co)induction

Fig. 1. Multi-Level Architecture

- More in general, there is a fruitful interaction between (co)-induction principles, Isabelle HOL datatypes, classical reasoning and hypothetical judgments, which tends to yield more automation than in a system such as Coq.

Our approach is also comparable with Twelf [21] (see Section [6), but is has a low mathematical overhead, as it simply consists of a package on top of Isabelle HOL. In a sense, we could look at Hybrid as a way to "compile" HOAS meta-proofs, such as Twelf's, into the well-understood setting of higher-order logic.

We demonstrate the method by formally verifying the correctness of (part of) a compiler for a fragment of Mini-ML, following [10]. To further exhibit the flexibility of our system, we modify the target language with a notion of nonwell-founded closure, inspired by Milner \& Tofte's paper [16] and formally verify, via co-induction, a subject reduction theorem for this new language.

The paper is organized as follows: in the next Section 2 we introduce at the informal level the syntax and semantics of our case study. Section 3 recalls some basics notion of Hybrid and its syntax-representing techniques. In Section 4 we introduce the multi-level architecture, while Section 5 is the heart of the paper, detailing the formal verification of compiler correctness. We conclude with a few words on related and future work (Section [6and 7).

We use a pretty-printed version of Isabelle HOL concrete syntax; a rule (a sequent) with conclusion $C$ and premises $H_{1} \ldots H_{n}$ will be represented as $\llbracket H_{1} ; \ldots ; H_{n} \rrbracket \Longrightarrow C$. An Isabelle HOL type declaration has the form $s::\left[t_{1}, \ldots t_{n}\right] \Rightarrow t$. Isabelle HOL connectives are represented via the usual logical notation. Free variables (upper-case) are implicitly universally quantified. The sign "=" (Isabelle meta-equality) is used for equality by definition, $\wedge$ for Isabelle universal meta-quantification. The keyword MC-Theorem denotes a machine-checked theorem, while (Co)Inductive introduces a (co)inductive relation in Isabelle HOL. We have tried to use the same notation for mathematical and formalized judgments. To facilitate the comparison with the Hannan \& Pfenning's approach, we have used the same nomenclature and convention as [18]. 


\section{The Case Study}

Compiler verification can be a daunting task, but it is a good candidate for mechanization. Restricting to functional languages (see [11] for issue related to compilation of Java for example), some first attempts were based on denotational semantics [3, 13]. Using instead operational semantics has the advantage that the meaning of both high and low level languages can be expressed in a common setting. In particular, operational semantics and program translations can be represented using deductive systems. This approach started in [4]1]. Other papers, for example [9], have explored aspects of higher-order encoding of abstract machines and compilation and [10] contains the first attempt to carry formal verification of compiler correctness in LF. Only recently the notion of regular world introduced in [21] provides a satisfying foundation for the theory of mode, termination and coverage checking [19, which has been developed to justify using LF for meta-reasoning.

We follow the stepwise approach to compilation in [10]; however, for reason of space, we limit ourselves to the verification of the correspondence between the big-step semantics of a higher-order functional language and the evaluation to closure of its translation into a target language of first-order expressions. For interest, though, we add a co-inductive twist due to Milner \& Tofte 16 in the treatment of fix points, which is analogous to the semantics of letrec in the original formulation of Mini-ML.

The language we utilize here is a strict lambda calculus augmented with a fix point operator, although it could be easily generalized to the rendition of Mini-ML in 18. This fragment is sufficient to illustrate the main ideas without cluttering the presentation with too many details. The types and terms of the source language are given respectively by:

$$
\begin{aligned}
& \text { Types } \tau::=\mathbf{i} \mid \tau_{1} \text { arrow } \tau_{2} \\
& \text { Terms } e::=x|\operatorname{lam} x . e| e @ e^{\prime} \mid \text { fix } x . e
\end{aligned}
$$

The rules for call-by-value operational semantics $(e \Downarrow v)$ and type inference $(\Gamma \vdash$ $e: \tau)$ are standard and are omitted — see Subsection 4.2 for the implementation. The usual subject reduction for this source language holds.

\subsection{Compilation}

We start the compilation process by translating the higher-order syntax into a first-order one. Terms are compiled into a simple calculus with explicit substitutions, where de Bruijn indexes are represented by appropriate shifting on the 1 numeral:

$$
d B \text { Terms } F::=1|F \uparrow| \mathbf{l a m}^{\prime} F\left|F @^{\prime} F^{\prime}\right| \mathbf{f i x}^{\prime} F
$$

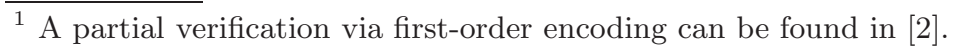


Then we introduce environments and closures:

$$
\begin{aligned}
\text { Environments } \quad \eta: & :=\cdot|\eta ; W| \eta+F \\
\text { Values } W & :=\{\eta, F\}
\end{aligned}
$$

In this setting the only possible values are closures, yet the presence of fixpoints requires environments to possibly contain unevaluated expressions, via the environment constructor ' + '. We will see in Subsection 2.2 how this can be refined using a notion of non-well-founded closure.

The operational semantics of this language (judgment $\eta \vdash F \hookrightarrow W$ ) uses environments to represent mappings of free variables to values and closures for terms whose free variables have values in the environment. We remark that due to the presence of unrestricted fix points, the rule fev_. $\mathbf{1}^{+}$in not just a look-up, but requires the evaluation of the body of the fix point.

$$
\begin{aligned}
& \frac{\eta \vdash F \hookrightarrow W}{\eta ; W \vdash 1 \hookrightarrow W} \text { fev_1 } \quad \frac{\eta \text { fev }_{-} \uparrow}{\eta ; W^{\prime} \vdash F \uparrow \hookrightarrow W} \\
& \frac{\eta \vdash F \hookrightarrow W}{\eta+F \vdash 1 \hookrightarrow W} \text { fev }_{\mathbf{1}} \mathbf{1}^{+} \quad \frac{\eta \vdash F \hookrightarrow W}{\eta+F^{\prime} \vdash F \uparrow \hookrightarrow W} \mathbf{f e v}_{-} \uparrow^{+} \\
& \frac{\eta \vdash \text { lam }^{\prime} F \hookrightarrow\left\{\eta, \text { lam }^{\prime} F\right\}}{\text { fev_lam }} \quad \frac{\eta+\text { fix }^{\prime} F \vdash F \hookrightarrow W}{\eta \vdash \text { fix }^{\prime} F \hookrightarrow W} \text { fev_fix }{ }^{\prime} \\
& \frac{\eta \vdash F_{1} \hookrightarrow\left\{\eta^{\prime}, \operatorname{lam}^{\prime} F_{1}^{\prime}\right\} \quad \eta \vdash F_{2} \hookrightarrow W_{2} \quad \eta^{\prime} ; W_{2} \vdash F_{1}^{\prime} \hookrightarrow W}{\eta \vdash F_{1} @^{\prime} F_{2} \hookrightarrow W} \text { fev_@ }^{\prime}
\end{aligned}
$$

The judgment $\eta \vdash F \leftrightarrow e$ (Figure 2) elegantly accomplishes the translation to the $\mathrm{dB}$ language using parametric and hypothetical judgments for the binding constructs: for the fix point, we assume we have extended the environment with a new expression parameter $f$; in the function case, the parameter $w$ ranges over values and the judgment is mutually recursive with value translation, $W \Leftrightarrow v$. As remarked in [18, this translation is total, but can be non-deterministic, when $\eta$ and $e$ are given and $F$ is computed.

The verification of compiler correctness can be graphically represented as:

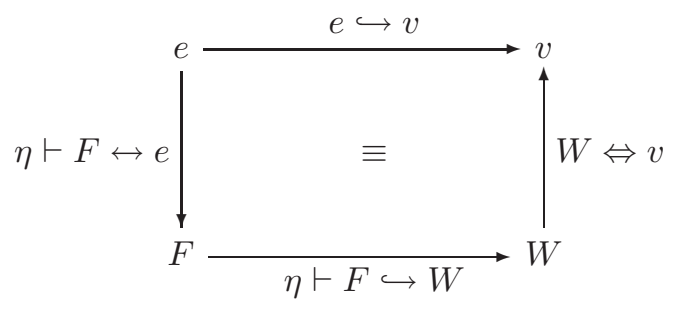

We discuss the statement and the proof once we provide the mechanization in Section 5$]$ 


$$
\begin{aligned}
& \overline{w \Leftrightarrow x}^{u} \quad \overline{\eta \vdash f \leftrightarrow x} u \\
& \frac{\eta ; w \vdash F \leftrightarrow e}{\eta \vdash \operatorname{lam}^{\prime} F \leftrightarrow \operatorname{lam} x . e}{\operatorname{tr} \_\operatorname{lam}^{w, x, u}}^{\frac{\eta+f \vdash F \leftrightarrow e}{\eta \vdash \mathbf{f i x}^{\prime} F \leftrightarrow \mathbf{f i x} x \cdot e}} \operatorname{tr} \_f i x^{f, x, u} \\
& \frac{W \Leftrightarrow e}{\eta ; W \vdash 1 \leftrightarrow e} \text { tr_1 } \quad \frac{\eta \vdash F \leftrightarrow e}{\eta ; W \vdash F \uparrow \leftrightarrow e} \operatorname{tr}_{-} \uparrow \\
& \frac{\eta \vdash F \leftrightarrow E}{\eta+F \vdash 1 \leftrightarrow e} \operatorname{tr}_{-} \mathbf{1}^{+} \quad \frac{\eta \vdash F \leftrightarrow e}{\eta+F^{\prime} \vdash F \uparrow \leftrightarrow e} \operatorname{tr}_{-} \uparrow^{+} \\
& \frac{\eta \vdash F_{1} \leftrightarrow e_{1} \quad \eta \vdash F_{2} \leftrightarrow e_{2}}{\eta \vdash F_{1} @^{\prime} F_{2} \leftrightarrow e_{1} @ e_{2}} \operatorname{tr} \_a p p \quad \frac{\eta \vdash \operatorname{lam}^{\prime} F \leftrightarrow \operatorname{lam} x . e}{\left\{\eta, \operatorname{lam}^{\prime} F\right\} \Leftrightarrow \operatorname{lam} x . e} \operatorname{vtr\_ lam}
\end{aligned}
$$

Fig. 2. Translation to modified dB terms and values

\subsection{Compilation via Non-Well-Founded Closures}

In order to illustrate the flexibility of our approach we now depart from [18] and draw inspiration from [16] to give a different and simplified treatment of fix points. First, we take up the SML-like restriction that the body of a fix point is always a lambda. Then the idea is simply to allow a fix point to evaluate to a "circular" closure, where, intuitively, the free variable points to the closure itself. This means swapping the following rule for fev_fix' ${ }^{\prime}$ and dropping rules fev_ $\mathbf{1}^{+}$ and $\mathbf{f e v}_{-} \uparrow^{+}$. The analogous rules in the translation are also dropped.

$$
\frac{c l=\left\{(\eta ; c l), \mathbf{l a m}^{\prime} F\right\}}{\eta \vdash \mathbf{f i x}^{\prime}\left(\operatorname{lam}^{\prime} F\right) \hookrightarrow c l} \mathbf{f e v \_ f i x} \mathbf{x}^{*}
$$

This amounts to a non-well-founded notion of closure. Other versions are possible, viz. recursive environments, see [4] for a discussion.

To exemplify the style of co-inductive reasoning entailed, we adapt the proof of subject reduction for closures in [16]. First we introduce typing for dB terms:

$$
\begin{gathered}
\frac{\Delta \vdash F: \tau}{\Delta ; \tau \vdash 1: \tau} \text { ftp_1 ftp_ } \uparrow \frac{\Delta ; \tau \vdash F: \tau^{\prime}}{\Delta ; \tau^{\prime} \vdash F \uparrow: \tau} \frac{\Delta \vdash \text { lam }^{\prime} F: \tau \text { arrow } \tau^{\prime}}{\text { ftp_lam }} \text { flam }^{\prime} \\
\frac{\Delta ; \tau \vdash F: \tau}{\Delta \vdash \text { fix }^{\prime} F: \tau} \text { ftp_fix } \\
\frac{\Delta \vdash F_{1}: \tau^{\prime} \text { arrow } \tau \quad \Delta \vdash F_{2}: \tau^{\prime}}{\Delta \vdash F_{1} @^{\prime} F_{2}: \tau} \text { ftp_@ }
\end{gathered}
$$

Typing of closures can be seen as taking the greatest fix point of the following rules, where we refer the reader to ibid. for motivation and examples: 


$$
\frac{\Delta \vdash \operatorname{lam}^{\prime} F: \tau \quad \eta:^{\prime} \Delta}{\left\{\eta, \operatorname{lam}^{\prime} F\right\}: \tau} \text { tpc } \quad \frac{W: \tau \quad \eta:^{\prime} \Delta}{\cdot !^{\prime} \cdot} \text { etp. } \quad \frac{W ;}{(\eta ; W) !^{\prime}(\Delta ; \tau)} \text {; }
$$

We note the "circularity" of rule tpc and etp; where the former requires welltypedness of value environment w.r.t. type environment.

Theorem 1 (Subject reduction for closures [16]). Let $\eta:^{\prime} \Delta$. If $\eta \vdash F \hookrightarrow W$ and $\Delta \vdash F: \tau$, then $W: \tau$.

\section{$3 \quad$ Hybrid Infrastructure}

We briefly recall that the theory Hybrid [1] provides support for a deep embedding of higher-order abstract syntax within Isabelle HOL. In particular, it provides a model of the untyped $\lambda$-calculus with constants. Let con denote a suitable type of constants. The model comprises a type expr together with functions

$$
\begin{array}{rr}
\text { CON }:: \text { con } \Rightarrow \exp r & \$ \$:: \operatorname{expr} \Rightarrow \exp r \Rightarrow \operatorname{expr} \\
\text { VAR }:: \text { nat } \Rightarrow \operatorname{expr} & \text { lambda }::(\exp r \Rightarrow \exp r) \Rightarrow \exp r
\end{array}
$$

and two predicates proper : : expr $\Rightarrow$ bool and abstr : : (expr $\Rightarrow$ expr $) \Rightarrow$ bool. The elements of expr which satisfy proper are in one-to-one correspondence with the terms of the untyped $\lambda$-calculus modulo $\alpha$-equivalence. The function CON is the inclusion of constants into terms, VAR is the enumeration of an infinite supply of free variables, and $\$ \$$ is application. The function lambda is declared as a binder and we write LAM $v$. $e$ for lambda $(\lambda v . e)$.

For this data to faithfully represent the syntax of the untyped $\lambda$-calculus, it must be that CON, VAR, $\$ \$$ are injective on proper expressions and furthermore, lambda is injective on some suitable subset of expr $\Rightarrow$ expr. This cannot the whole of $\operatorname{expr} \Rightarrow \operatorname{expr}$ for cardinality reasons. In fact, we need only a small fragment of the set. The predicate abstr identifies those functions which are sufficiently parametric to be realized as the body of a $\lambda$-term, and lambda is injective on these. This predicate can be seen as a full HOAS counterpart of the valid $_{1}$ judgment in [5], but it must be defined at the de Bruijn level, since a higher-order definition would require a theory of $n$-ary abstractions, which is the object of current research.

There is a strong tradition in the HOL community of making extensions by definition wherever possible. This ensures the consistency of the logic relative to a small axiomatic core. Hybrid is implemented in a definitional style using a translation into de Bruijn notation. The type expr is defined by the grammar

$$
\text { expr }:=\text { CON con | VAR var | BND bnd | expr } \$ \$ \text { expr | ABS expr }
$$

The translation of terms is best explained by example. Let $T_{O}=\Lambda V_{1} \cdot \Lambda V_{2} \cdot V_{1} V_{3}$ be an expression in the concrete syntax of the $\lambda$-calculus. This is rendered in Hybrid as $T_{H}=\mathrm{LAM} v_{1}$. (LAM $v_{2} \cdot\left(v_{1} \$ \$\right.$ VAR 3$\left.)\right)$ - note the difference between the treatment of free variables and of bound variables. Expanding the binder, 
this expression is by definition lambda $\left(\lambda v_{1}\right.$. (lambda $\lambda v_{2} \cdot\left(v_{1} \$ \$\right.$ VAR 3$\left.\left.)\right)\right)$, where $\lambda v_{i}$ is meta-abstraction. The function lambda : : (expr $\left.\Rightarrow \operatorname{expr}\right) \Rightarrow \exp r$ is defined so as to map any function satisfying abstr to a corresponding proper de Bruijn expression. Again, it is defined as an inductive relation on the underlying representation and then proven to be functional. The expression $T_{H}$ is reduced by higher-order rewriting to the de Bruijn term ABS (ABS (BND $1 \$ \$$ VAR 3)). Given these definitions, the essential properties of Hybrid expressions can be proved as theorems from the properties of the underlying de Bruijn representation: for instance, the injectivity of lambda

$$
\llbracket \operatorname{abstr} E ; \operatorname{abstr} F \rrbracket \Longrightarrow(\operatorname{LAM} v \cdot E v=\operatorname{LAM} v \cdot F v)=(E=F) \quad I N J
$$

and extensionality $\llbracket \operatorname{abstr} E ; \operatorname{abstr} F ; \forall i . E(\operatorname{VAR} i)=F(\operatorname{VAR} i) \rrbracket \Longrightarrow E=F$. Several principles of induction over proper expressions are also provable.

\subsection{Coding the Syntax of OL System in Hybrid}

We begin by showing how to represent (a fragment of) Mini-ML in Hybrid. In order to render the syntax of the source language in HOAS format we need constants for abstraction, application and fix point, say $c A P P, c A B S$ and $c F I X$. Recall that in the meta-language application is denoted by infix $\$ \$$, and abstraction by LAM. Then the source language corresponds to the grammar:

$$
e::=v|c A B S \$ \$(\operatorname{LAM} v \cdot e v)| c A P P \$ \$ e_{1} \$ \$ e_{2} \mid c F I X \$ \$(\operatorname{LAM} v . e v)
$$

Thus, we declare these constants to belong to con and then make the following definitions:

$$
\begin{aligned}
& \text { @ : : }[\exp , \exp ] \Rightarrow \exp \quad \text { lam }::(\exp \Rightarrow \exp ) \Rightarrow \exp \\
& e_{1} @ e_{2}=\operatorname{CON} c A P P \$ \$ e_{1} \$ \$ e_{2} \quad \operatorname{lam} x . E x=\operatorname{CON} c A B S \$ \$ \text { LAM } x . e E
\end{aligned}
$$

and similarly for the fix point, where lam (resp. fix) is indeed an Isabelle HOL binder. For example, the "real" underlying form of fix $x \cdot \operatorname{lam} y . x @ y$ is

$$
\text { CON } c F I X \$ \$(\operatorname{LAM} x . \operatorname{CON} c A B S \$ \$ \operatorname{LAM} y \cdot(\operatorname{CON} c A P P \$ \$ x \$ \$ y)
$$

It is now possible to prove the freeness properties of constructors.

MC-Theorem 1. The constructors have distinct images; for example, lam $x . E x \neq$ fix $x . E^{\prime} x$. Furthermore, every binding constructor is injective on abstractions; for example, $\llbracket$ abstr $E$; abstr $E^{\prime} \rrbracket \Longrightarrow($ fix $x . E x=$ fix $\left.x . E^{\prime} x\right)=\left(E=E^{\prime}\right)$.

This is proven via Isabelle HOL's simplification, using property INJ.

Although dB expressions are strictly first-order, we still need to encode them as Hybrid expressions. In fact, we will use for compilation a judgment which is parametric in (higher-order) terms, dB expression and values. Therefore they all have to be interpreted via the SL universal quantification and consequently need 
to be synonymous to Hybrid (proper) terms, to make the universal quantification consistent2. The encoding is trivial and the details omitted.

The informal definition of environments and closure is by mutual recursion. Since our aim here is also to show how Hybrid expressions can coexist with regular Isabelle HOL ones, we will use Isabelle HOL mechanism for mutually inductive datatypes. This brings about the declaration of a datatype of polymorphic environments, intended to be instantiated with a value. Environments can be now mutually recursive with closures, where the type synonymous $\exp ^{\prime}$ is retained for forward compatibility with Subsection 5.1

$$
\begin{aligned}
\text { datatype }(\alpha \text { env })::=\cdot \mid(\alpha \text { env }) ; \alpha \mid(\alpha \text { env })+\exp ^{\prime} \\
\text { and } \alpha \text { clos }::=\mathrm{mk}_{\mathbf{n}} \text { clo }(\alpha \text { env }) \exp ^{\prime}
\end{aligned}
$$

Then we introduce in con a constructor, say $c C L O($ val clos $)$, which encapsulates a Isabelle HOL closure. Finally, we can define a Hybrid closure as a constant of type $\left[(v a l e n v), e x p^{\prime}\right] \Rightarrow v a l$, defined as $\{\eta, F\}=\operatorname{CON}(c C L O($ mk_clo $\eta F))$. Thanks to this rather cumbersome encoding, we can establish freeness properties of closures as in Theorem[1.

\section{Multi-level Architecture}

In previous work [1, 17], we chose to work in a single level, implementing every judgment as a (co)inductive definition in Isabelle HOL, but exploiting the form of HOAS that our package supports. While the tool seemed successful in dealing with predicates over closed terms, say evaluation, we had to resort to a more traditional encoding, i.e. via explicit environments, with respect to judgments involving open ones such as typing. As we have mentioned earlier, a two-level approach solves this dilemma.

\subsection{Encoding the Specification Logic}

We introduce our specification logic, namely a fragment of second-order hereditary Harrop formulae, which is sufficient for the encoding of our case-study.

$$
\begin{array}{r}
\text { Clauses } D::=A \mid D_{1} \text { and } D_{2} \mid G \text { imp } A|\mathrm{tt}| \text { all } x . D \\
\text { Goals } G::=A \mid G_{1} \text { and } G_{2}|A \operatorname{imp} G| \mathrm{tt} \mid \text { all } x . G
\end{array}
$$

This syntax translates immediately in a Isabelle HOL datatype:

$$
\text { datatype oo }::=\mathrm{tt}|\langle a \mathrm{tm}\rangle| \text { oo and oo } \mid \text { atm imp oo } \mid \text { all }(\mathrm{prpr} \Rightarrow \text { oo })
$$

where $\left\langle_{-}\right\rangle$coerces atoms into propositions. The universal quantifier is intended to range over all proper Hybrid terms. In analogy with logic programming, it will be left implicit in clauses.

\footnotetext{
${ }^{2}$ This is because Hybrid, in its current formulation, provides only what amounts to an untyped meta-language. This is being addressed in the next version
} 
This logic is so simple that its proof-system can be modeled with a logic programmer interpreter; in fact, for such a logic, uniform provability [15] (of a sequent-calculus) is complete. We give the following definition of provability:

$$
\begin{aligned}
\text { Inductive _ } \triangleright_{-}{ }_{-} & :[\text {nat },(\text { atmlist }), \text { oo }] \Rightarrow \text { bool } \\
& \Longrightarrow \Phi \triangleright_{n} \text { tt } \\
\llbracket \Phi \triangleright_{n} G_{1} ; \Phi \triangleright_{n} G_{2} \rrbracket & \Longrightarrow \Phi \triangleright_{n+1}\left(G_{1} \text { and } G_{2}\right) \\
\llbracket \forall x . \Phi \triangleright_{n}(G x) \rrbracket & \Longrightarrow \Phi \triangleright_{n+1}(\text { all } x . G x) \\
\llbracket \Phi, A \triangleright_{n} G \rrbracket & \Longrightarrow \Phi \triangleright_{n+1}(A \mathrm{imp} G) \\
\llbracket A \in \Phi \rrbracket & \Longrightarrow \Phi \triangleright_{n}\langle A\rangle \\
\llbracket A \longleftarrow G ; \Phi \triangleright_{n} G \rrbracket & \Longrightarrow \Phi \triangleright_{n+1}\langle A\rangle
\end{aligned}
$$

Note the following:

- Only atomic antecedent are required in implications which therefore yield only atomic contexts.

- Atoms are provable either by assumptions or via backchaining over a set of Prolog-like rules, which encode the properties of the object-logic in question. The suggestive notation $A \longleftarrow G$ corresponds to an inductive definition of a set prog of type $[\mathrm{atm}, o \mathrm{o}] \Rightarrow$ bool, see Subsection 4.2 The sequent calculus is parametric in those clauses and so are its meta-theoretical properties.

- Sequents are decorated with natural numbers which represent the height of a proof; this measure allows reasoning by complete induction.

- For convenience we define $\Phi \triangleright G$ iff $\exists n . \Phi \triangleright_{n} G$ and $\triangleright G$ iff $\cdot \triangleright G$.

- The very fact that provability is inductive makes available inversion principles as elimination rules of the aforementioned definition. In Isabelle HOL (as well as in Coq) case analysis is particularly well-supported as part of the datatype/inductive package. For example the inversion theorem that analyses the shape of a derivation ending in an atom from the empty context is obtained simply with a call to the built-in mk_cases function, which specializes the elimination principle to the given constructors:

$$
\llbracket \cdot \triangleright_{i}\langle A\rangle ; \bigwedge G j \cdot \llbracket A \longleftarrow G ; \cdot \triangleright_{j} G ; i=j+1 \rrbracket \Longrightarrow P \rrbracket \Longrightarrow P
$$

- The adequacy of the encoding of the SL can be established as in [14].

MC-Theorem 2 (Structural Rules). The following rules are provable:

1. Weakening for numerical bounds: $\Phi \triangleright_{n} G ; n<m \rrbracket \Longrightarrow \Phi \triangleright_{m} G$

2. Context weakening: $\llbracket \triangleright G ; \Phi \subseteq \Phi^{\prime} \rrbracket \Longrightarrow \Phi^{\prime} \triangleright G$

3. (Atomic) cut: $\Phi, A \triangleright G ; \Phi \triangleright\langle A\rangle \rrbracket \Longrightarrow \Phi \triangleright G$

Proof.

1. The proof, by structural induction on sequents, consists of a one-line call to an automatic tactic using the elimination rule for successor (from the Isabelle HOL library) and the introduction rules for the sequent calculus. This compared to the much longer proof of the same statement in Coq reported in [6]. 
2. By a similar fully automated induction on the structure of the sequent derivation.

3. Cut is a corollary of the following lemma:

$$
\llbracket \Phi^{\prime} \triangleright_{i} G ; \Phi^{\prime}=\operatorname{set}(\Phi, A) ; \Phi \triangleright_{j}\langle A\rangle \rrbracket \Longrightarrow \Phi \triangleright_{i+j} G
$$

easily proven by induction on the structure of the derivation of $\Phi^{\prime} \triangleright_{i} G$, using library facts relating set and list memberships.

\subsection{Encoding the Object Logic}

We introduce a datatype atm to encode the atomic formulae of the OL, which in this case study includes

$$
\text { datatype atm ::= exp : tp }|\exp \Downarrow \exp |(\text { val env }) \vdash \exp p^{\prime} \leftrightarrow \exp \mid \text { val } \Leftrightarrow \exp
$$

We can now give the clauses for the OL deductive systems; we start with typing and evaluation:

$$
\begin{aligned}
& \text { Inductive _ } \longleftarrow::[\text { atm }, \text { oo }] \Rightarrow \text { bool } \\
& \Longrightarrow\left(E_{1} @ E_{2}\right): T \longleftarrow\left\langle E_{1}:\left(T^{\prime} \text { arrow } T\right)\right\rangle \text { and }\left\langle E_{2}: T^{\prime}\right\rangle \\
& \llbracket \operatorname{abstr} E \rrbracket \Longrightarrow(\operatorname{lam} x . E x):\left(T_{1} \text { arrow } T_{2}\right) \longleftarrow \text { all } x .\left(x: T_{1}\right) \operatorname{imp}\left\langle(E x): T_{2}\right\rangle \\
& \llbracket \operatorname{abstr} E \rrbracket \Longrightarrow(\text { fix } x . E x): T \longleftarrow \text { all } x .(x: T) \operatorname{imp}\langle(E x): T\rangle \\
& \llbracket \operatorname{abstr} E \rrbracket \Longrightarrow \operatorname{lam} x . E x \Downarrow \operatorname{lam} x . E x \longleftarrow \text { tt } \\
& \llbracket \text { abstr } E_{1}^{\prime} \rrbracket \Longrightarrow E_{1} @ E_{1} \Downarrow V \longleftarrow \\
& \left\langle E_{1} \Downarrow \operatorname{lam} x . E_{1}^{\prime} x\right\rangle \text { and }\left\langle E_{2} \Downarrow V_{2}\right\rangle \text { and }\left\langle\left(E_{1}^{\prime} V_{2}\right) \Downarrow V\right\rangle \\
& \llbracket \operatorname{abstr} E \rrbracket \Longrightarrow \text { fix } x . E x \Downarrow V \longleftarrow\langle E(\text { fix } x . E x) \Downarrow V\rangle
\end{aligned}
$$

Note the presence of the abstraction annotations as Isabelle HOL premises in rules mentioning binding construct. This in turn allows to simulate definitional reflection via the built-in elimination rules of the prog inductive definition without the use of additional axioms. For example inversion on the function typing rule is:

$\left.\llbracket \operatorname{lam} x . E x: \tau \longleftarrow G ; \bigwedge F, T_{1}, T_{2} . \llbracket \operatorname{abstr} F ; G=\operatorname{all} x .\left(x: T_{1}\right) \operatorname{imp}\left\langle F x: T_{2}\right\rangle\right)$ lambda $E=$ lambda $F ; \tau=\left(T_{1} \rightarrow T_{2}\right) \rrbracket \Longrightarrow P \rrbracket \Longrightarrow P$

Note also how the inversion principle has an explicit equation lambda $E=$ lambda $F$ (whereas definitional reflection employs unification) and those are solvable under the assumption that the body of a lambda term is well-behaved, i.e. an abstraction.

Now we can address the meta-theory, starting, for example, with the subject reduction theorem:

MC-Theorem 3 (OL Subject Reduction).

$\forall n .\left(\cdot \triangleright_{n} E \Downarrow V\right) \Longrightarrow \forall T .(\triangleright E: T) \rightarrow(\triangleright V: T)$. 
Proof. The proof is by complete induction on the height of the derivation of evaluation, analogously to [6] (except with an appeal to Theorem 1 rather than to the distinctness axioms).

As we remarked, the main reason to reference provability is the intrinsic incompatibility of induction with hypothetical (non-stratifiable) judgments. Since the definition of evaluation makes no use of hypothetical judgments, it is perfectly acceptable at the meta-level, that is, we can directly give an inductive definition for it.

$$
\begin{aligned}
& \text { Inductive _ } \Downarrow \_:: \quad[\exp , \exp ] \Rightarrow \text { bool } \\
& \llbracket E_{1} \Downarrow \operatorname{lam} x . E^{\prime} x ; \text { abstr } E^{\prime} ; \\
& E_{2} \Downarrow V_{2} ;\left(E^{\prime} V_{2}\right) \Downarrow V \rrbracket \Longrightarrow\left(E_{1} @ E_{2}\right) \Downarrow V \\
& \llbracket \operatorname{abstr} E \rrbracket \Longrightarrow \operatorname{lam} x . E x \Downarrow \operatorname{lam} x . E x
\end{aligned}
$$

$\llbracket \operatorname{abstr} E ;(E($ fix $x . E)) \Downarrow V \rrbracket \Longrightarrow$ fix $x . E \Downarrow V$

Moreover, it is easy to show (formally) the two encodings equivalent:

MC-Theorem 4. $E \Downarrow V$ iff $\cdot \triangleright_{n} E \Downarrow V$.

Proof. Left-to-right holds by structural induction. The converse is by complete induction.

This suggest we delegate to the OL level only those judgments, such as typing, which would not be inductive at the meta-level. This has the benefit of limiting the indirect-ness of using an explicit SL. Moreover, it has the further advantage of replacing complete with structural induction, which is better behaved from a proof-search point of view. Complete induction, in fact, places an additional burden to the user by requiring him/her to provide the correct instantiation for the height of the derivation in question. Thus subject reduction at the meta-level has the form:

MC-Theorem 5 (Meta-level Subject Reduction). $E \Downarrow V \Longrightarrow \forall T$. $(\triangleright E: T) \rightarrow(\triangleright V: T)$.

Proof. By structural induction on evaluation, using only inversion principles on provability and OL typing.

\section{Formal Verification of Compilation}

Similarly, we implement evaluation to closures $\eta \vdash F \hookrightarrow W$ at the meta-level as a straightforward (i.e. entirely first-order) inductive definition, whose rules we omit. Compilation to $\mathrm{dB}$ expressions and values is instead represented at the OL level.

$$
\begin{gathered}
\Longrightarrow \eta \vdash\left(F_{1} @^{\prime} F_{2}\right) \leftrightarrow\left(E_{1} @ E_{2}\right) \longleftarrow \\
\left\langle\eta \vdash F_{1} \leftrightarrow E_{1}\right\rangle \text { and }\left\langle\eta \vdash F_{2} \leftrightarrow E_{2}\right\rangle \\
\llbracket \text { abstr } E \rrbracket \Longrightarrow \eta \vdash \operatorname{lam}^{\prime} F \leftrightarrow(\operatorname{lam} x . E x) \longleftarrow \\
\text { all } w \text {. all } x . w \Leftrightarrow x \operatorname{imp}\langle(\eta ; w) \vdash F \leftrightarrow(E x)\rangle
\end{gathered}
$$




$$
\begin{aligned}
& \llbracket \operatorname{abstr} E \rrbracket \Longrightarrow \eta \vdash \text { fix }^{\prime} F \leftrightarrow(\text { fix } x . E x) \longleftarrow \\
& \text { all } f \text {. all } x . \eta \vdash f \leftrightarrow x \operatorname{imp}\langle(\eta+f) \vdash F \leftrightarrow(E x)\rangle \\
& \Longrightarrow(\eta ; W) \vdash 1 \leftrightarrow E \longleftarrow\langle W \Leftrightarrow E\rangle \\
& \Longrightarrow(\eta ; W) \vdash F \uparrow \leftrightarrow E \longleftarrow\langle\eta \vdash F \leftrightarrow E\rangle \\
& \llbracket \operatorname{abstr} E \rrbracket \Longrightarrow\left\{\eta, \operatorname{lam}^{\prime} F\right\} \Leftrightarrow \operatorname{lam} x . E x \longleftarrow\left\langle\eta \vdash \operatorname{lam}^{\prime} F \leftrightarrow \operatorname{lam} x . E x\right\rangle
\end{aligned}
$$

We can now tackle the verification of compiler correctness:

\section{MC-Theorem 6 (Soundness of Compilation).}

$E \Downarrow V \Longrightarrow \forall n \eta F .\left(\cdot \triangleright_{n} \eta \vdash F \leftrightarrow E\right) \rightarrow \exists W . \eta \vdash F \hookrightarrow W \wedge(\triangleright W \Leftrightarrow V)$

Proof. The informal proof proceeds by lexicographic induction on the pair consisting of the derivation $\mathcal{D}$ of $E \Downarrow V$ and $\mathcal{C}$ of $\eta \vdash F \leftrightarrow E$. We examine one case, both formally and informally to demonstrate the use of hypothetical judgments and its realization in the system. Let $\mathcal{D}$ end in ev_app and assume $\eta \vdash F \leftrightarrow e_{1} @ e_{2}$. Inversion on the latter yields three cases. Let's consider only the last one, where $F=F_{1} @^{\prime} F_{2}$ such that $\eta \vdash F_{1} \leftrightarrow e_{1}$ and $\eta \vdash F_{2} \leftrightarrow e_{2}$. By IH, $\eta \vdash F_{2} \hookrightarrow W_{2}$ and $W_{2} \Leftrightarrow v_{2}$. Moreover, $\eta \vdash F_{1} \hookrightarrow W_{1}$ and $W_{1} \Leftrightarrow \operatorname{lam} x$. $e_{1}^{\prime}$. By inversion, $W_{1}=\left\{\eta_{1}, \operatorname{lam}^{\prime} F_{1}^{\prime}\right\}$ and $\eta_{1} \vdash \operatorname{lam}^{\prime} F_{1}^{\prime} \leftrightarrow \operatorname{lam} x . e_{1}^{\prime}$. By a further inversion we have a proof of $\eta_{1} ; w \vdash F_{1}^{\prime} \leftrightarrow e_{1}^{\prime}$ under the parametric assumption $w \Leftrightarrow x$. Now substitute $W_{2}$ for $w$ and $v_{2}$ for $x$ : since $W_{2} \Leftrightarrow v_{2}$ holds, we infer $\eta_{1} ; W_{2} \vdash F_{1}^{\prime} \leftrightarrow\left[v_{2} / x\right] e_{1}^{\prime}$. We can now apply the IH once more yielding $\eta_{1} ; W_{2} \vdash F_{1}^{\prime} \hookrightarrow W_{3}$ and $W_{3} \Leftrightarrow v$. The case is finished with an appeal to rule feval_app.

In the formal development we proceed by a nested induction, the outermost on the structure of $E \Downarrow V$ and the innermost by complete induction on $n$, representing the height of the SL's proof of $\eta \vdash F \leftrightarrow E$. Let

$I H(E, V)=\forall n \eta F . \triangleright_{n}\langle\eta \vdash F \leftrightarrow E\rangle \rightarrow \exists W . \eta \vdash F \hookrightarrow W \wedge \triangleright\langle W \Leftrightarrow V\rangle$ and $G=\exists W . \eta \vdash F_{1} @^{\prime} F_{2} \hookrightarrow W \wedge \triangleright\langle W \Leftrightarrow V\rangle$. Then the case for application from the outermost induction is, omitting the innermost IH which is not used here:

$\llbracket E_{1} \Downarrow \operatorname{lam} x . E^{\prime} x ; I H\left(E_{1}, \operatorname{lam} x . E_{1}^{\prime} x\right) ; \operatorname{abstr} E^{\prime} ; E_{2} \Downarrow V_{2} ; I H\left(E_{2}, V_{2}\right) ;$

$\left(E^{\prime} V_{2}\right) \Downarrow V ; I H\left(\left(E^{\prime} V_{2}\right), V\right) ; \cdot \triangleright_{n}\left\langle\eta \vdash F_{1} \leftrightarrow E_{1}\right\rangle$ and $\left\langle\eta \vdash F_{2} \leftrightarrow E_{2}\right\rangle \rrbracket \Longrightarrow G$

Eliminating the SL conjunction "and" and applying twice the IH, we get:

$\llbracket \ldots \exists W . \eta \vdash F_{1} \hookrightarrow W \wedge \triangleright\left\langle W \Leftrightarrow \operatorname{lam} x . E^{\prime} x\right\rangle ; \exists W . \eta \vdash F_{2} \hookrightarrow W \wedge \ldots \rrbracket \Longrightarrow G$

Now we perform inversion on $W \Leftrightarrow \operatorname{lam} x . E^{\prime} x$ and simplification:

$$
\llbracket \ldots \triangleright\left\langle\eta \vdash \operatorname{lam}^{\prime} F_{1}^{\prime} \leftrightarrow \operatorname{lam} x . E_{1}^{\prime} x\right\rangle \ldots \rrbracket \Longrightarrow G
$$

More inversion on $\eta_{1} \vdash \operatorname{lam}^{\prime} F_{1}^{\prime} \leftrightarrow \operatorname{lam} x . E_{1}^{\prime} x$, plus Hybrid injectivity to solve lambda $E=$ lambda $E_{1}^{\prime}$ :

$$
\begin{gathered}
\llbracket \ldots \eta \vdash F_{1} \hookrightarrow\left\{\eta_{1}, \operatorname{lam}^{\prime} F_{1}^{\prime}\right\} ; \eta \vdash F_{2} \hookrightarrow W_{2} ; \triangleright\left\langle W_{2} \Leftrightarrow V_{2}\right\rangle ; \\
\quad \text { all } w \text {. all } x .\left(w \Leftrightarrow x \operatorname{imp}\left\langle\eta_{1} ; w \vdash F_{1}^{\prime} \leftrightarrow\left(E_{1}^{\prime} x\right)\right\rangle\right) \rrbracket \Longrightarrow G
\end{gathered}
$$


We now invert on the (proof of the) parametric and hypothetical judgment and then instantiate $w$ with $W_{2}$ and $x$ with $V_{2}$ :

$$
\begin{aligned}
\llbracket \ldots \eta \vdash F_{1} \hookrightarrow & \left\{\eta_{1}, \operatorname{lam}^{\prime} F_{1}^{\prime}\right\} ; \eta \vdash F_{2} \hookrightarrow W_{2} ; \triangleright\left\langle W_{2} \Leftrightarrow V_{2}\right\rangle ; \\
& W_{2} \Leftrightarrow V_{2} \triangleright\left\langle\eta_{1} ; W_{2} \vdash F_{1}^{\prime} \leftrightarrow\left(E_{1}^{\prime} V_{2}\right)\right\rangle \rrbracket \Longrightarrow G
\end{aligned}
$$

After a cut we can apply the IH to $\eta_{1} ; W_{2} \vdash F_{1}^{\prime} \leftrightarrow\left(E_{1}^{\prime} V_{2}\right)$ yielding $\eta_{1} ; W_{2} \vdash$ $F_{1}^{\prime} \hookrightarrow W$. Then the proof is concluded with the introduction rule for application.

The converse does not contribute any new ideas and we leave it to the on-line documentation:

MC-Theorem 7 (Completeness of Compilation). $\eta \vdash F \hookrightarrow W \Longrightarrow \forall n E .\left(\cdot \triangleright_{n} \eta \vdash F \leftrightarrow E\right) \rightarrow \exists V . E \Downarrow V \wedge \triangleright W \Leftrightarrow V$

Proof. By structural induction on $\eta \vdash F \hookrightarrow W$ and inversion on $\cdot \triangleright_{n} \eta \vdash F \leftrightarrow E$.

\subsection{Implementation of Subject Reduction for Closures}

We now turn to the co-inductive part. Ideally, we would implement closures and environment as a co-datatype; indeed, this is possible in Isabelle/ZF, but not at the moment in Isabelle HOL. We then resort to a definitional approach where we introduce a Hybrid constant $\left\{\boldsymbol{-}_{-}\right\}^{\infty}::\left[(\right.$ val env $\left.), e x p^{\prime}\right] \Rightarrow$ val and we prove it to be injective as usual. There are alternatives; for example we have implemented on operational semantics with recursive closures, but we present here the above to be faithful to [16]. On the other hand, as remarked in Subsection 2.2, since we will not need the + environment constructor anymore, nothing prevents us from encoding here $\mathrm{dB}$ expressions with a Isabelle HOL datatype:

$$
\text { datatype } \exp ^{\prime}::=1\left|\exp p^{\prime} \uparrow\right| \mathbf{l a m}^{\prime} \exp ^{\prime}\left|\exp ^{\prime} @^{\prime} \exp ^{\prime}\right| \mathrm{fix}^{\prime} \exp ^{\prime}
$$

We only mention the new "circular" rule:

$$
\begin{aligned}
\text { Inductive } \_\vdash{ }_{-} \hookrightarrow{ }_{-}:: & {\left[(\text {val env }), \exp ^{\prime}, v a l\right] \Rightarrow \text { bool } } \\
\llbracket c l=\left\{(\eta ; c l), \mathbf{l a m}^{\prime} F\right\}^{\infty} \rrbracket & \Longrightarrow \eta \vdash \mathbf{f i x}^{\prime}\left(\mathbf{l a m}^{\prime} F\right) \hookrightarrow c l
\end{aligned}
$$

We declare a standard HOL datatype for types environments tenv and we encode the judgment $\Delta \vdash F: \tau$ with an inductive definitions of type $\llbracket$ tenv, exp ${ }^{\prime}, t p \rrbracket \Rightarrow$ bool, whose rules are obvious and again omitted. More interestingly, we introduce typing of closures and type consistency of value and type environments as a mutually co-inductive definition:

$$
\begin{aligned}
& \text { Coinductive _ : _ }:: \quad[v a l, t p] \Rightarrow \text { bool } \\
& \text { _ }:^{\prime} \text { _ }::[(\text { val env }), \text { tenv }] \Rightarrow \text { bool } \\
& \llbracket \Delta \vdash \operatorname{lam}^{\prime} F: \tau ; \eta:^{\prime} \Delta \rrbracket \Longrightarrow\left\{\eta, \operatorname{lam}^{\prime} F\right\}^{\infty}: \tau \\
& \Longrightarrow \cdot:^{\prime} \text {. } \\
& \llbracket W: \tau ; \eta:^{\prime} \Delta \rrbracket \Longrightarrow(\eta ; W):^{\prime}(\Delta ; \tau)
\end{aligned}
$$


MC-Theorem 8. $\eta \vdash F \hookrightarrow W \Longrightarrow \forall T . \eta:^{\prime} \Delta \rightarrow(\Delta \vdash F: \tau \rightarrow W: \tau)$

Proof. By structural induction on evaluation, where each case is proven with an appeal to an automatic tactic, which uses appropriate elimination rules. The only delicate case is the fix point, where we need to prove:

$$
\llbracket c l=\left\{(\eta ; c l), \operatorname{lam}^{\prime} F\right\}^{\infty} ; \eta:^{\prime} \Delta ; \Delta \vdash \operatorname{fix}^{\prime}\left(\operatorname{lam}^{\prime} F\right): \tau \rrbracket \Longrightarrow c l: \tau
$$

In Isabelle HOL (co)induction is realized set theoretically via the KnasterTarski's construction and the user provides the right set to be checked for density w.r.t. the rule set. Since our definition is by mutual co-induction, the greatest fix point is constructed as a disjoint sum. Thus, the right set turns out to be $\{\operatorname{lnr}(\eta ; c l), \operatorname{lnl}(\Delta ; \tau)\}$.

\section{Related Work}

We have so far concentrated on $F O \lambda^{\Delta I N}$, but the other major contender in the field is the Twelf project 20]. Meta-reasoning can be carried over in two complementary styles. In an interactive one [19], LF is used to specify a meta-theorem as a relation between judgments, while a logic programming-like interpretation provides the operational. Finally, external checks (termination, moded-ness, totality) verify that the given relation is indeed a realizer for that theorem. The second approach 21 is built on the idea of devising an explicit meta-meta-logic for reasoning (inductively) about logical frameworks, in a fully automated way. $\mathcal{M}_{\omega}$ is a constructive first-order logic, whose quantifiers range over possibly open LF object over a signature. By the adequacy of the encoding, the proof of the existence of the appropriate LF object(s) guarantees the proof of the corresponding object-level property. It must be remarked that Twelf is not programmable by tactics, nor does it support co-induction.

Other architectures are essentially one level. For lack of space, we refer to the review in [1], but we just mention Honsell et al.'s framework 12, which embraces an axiomatic approach to meta-reasoning with HOAS. It consists of higher-order logic extended with a set of axioms parametric to a HOAS signature, including the reification of key properties of names akin to freshness. A limited form of recursion over HOAS syntax is also assumed. Similarly the FM approach [7] aims to be a foundation of programming and reasoning with names in a onelevel architecture. It would be interesting to look at using a version of the "New" quantifier in the specification logic, especially for those applications where the behavior of the object-logic binder is not faithfully mirrored by a traditional universal quantification at the SL-level, for example the $\pi$-calculus.

\section{Conclusions and Future Work}

We have presented a multi-level architecture to allow (co)inductive reasoning about objects defined via HOAS in a well-known environment such as Isabelle HOL. Similarly to [6] this has several benefits: 
- It is possible to replicate in an well-understood and interactive setting the style of proof of $F O \lambda^{\Delta I N}$, so all results are proven without "technical" lemmas foreign to the mathematics of the problem.

- Results about the specification logic, such as cut elimination, are proven once and for all, if we are happy with that logic. Otherwise, different logics (say linear) can be employed, without changing infrastructure. This would allow, for example, the utilization of the most elegant encodings of the meta-theory of functional programming with references proposed, for instance, in [14].

Differently to [6], our architecture is based not directly on a standard proofassistant, but on a package which builds a HOAS meta-language on top of such a system. This allows us not to rely on any axiomatic assumptions, such as freeness of HOAS constructors and extensionality properties at higher types, which are now theorems. Another difference is the mixing of meta-level and OL specifications, which we have shown makes proofs more easily mechanizable and allows us to use co-induction which is still unaccounted for in $F O \lambda^{\Delta N}$. Finally, by the simple reason that the Hybrid system sits on top of Isabelle HOL, we benefit of the higher degree of automation of the latter.

As far as future work is concerned, we plan to further pursue our case study by finally compiling our target language into a CAM-like abstract machine, as in [10. We shall also investigate co-inductive issues in compilation, starting with verifying the equivalence between the standard operational semantics and the one with non-well founded closures.

Note that in this case study we only needed to induct - either directly, or on the height of derivations - over closed terms, although we extensively reasoned in presence of hypothetical judgments. Inducting HOAS-style over open terms is a major challenge 21]; in this setting generic judgments are particularly problematic, but can be dealt with by switching to a more expressive SL, based on a eigenvariable encoding [14. While it is already simple enough to implement such a logic, the new theory of $n$-ary abstractions which underlines the next version of the Hybrid infrastructure will directly support this syntax, as well as a form of Isabelle HOL-like datatypes over HOAS signatures. With that in place, we will be able, for example, to revisit in a full HOAS style the material in [17.

Source files for the Isabelle HOL code can be found at www.mcs.le.ac.uk/ amomigliano/isabelle/2Levels/Compile/main.html

Acknowledgments This paper has benefited from referees comments and discussions with Roy Crole, Dale Miller, Frank Pfenning, Carsten Schürmann and Amy Felty, who kindly made available to us the Coq proof script of [6].

\section{References}

[1] S. Ambler, R. Crole, and A. Momigliano. Combining higher order abstract syntax with tactical theorem proving and (co)induction. In V. A. Carreño, editor, Proceedings of the 15th International Conference on Theorem Proving in Higher Order Logics, volume 2342 of LNCS. Springer Verlag, 2002. 
[2] S. Boutin. Proving correctness of the translation from mini-ML to the CAM with the Coq proof development system. Technical Report RR-2536, Inria, Institut National de Recherche en Informatique et en Automatique, 1995.

[3] B. Ciesielski and M. Wand. Using the theorem prover Isabelle-91 to verify a simple proof of compiler correctness. Technical Report NU-CCS-91-20, College of Computer Science, Northeastern University, Dec. 1991.

[4] J. Despeyroux. Proof of translation in natural semantics. In Proceedings of LICS'86, pages 193-205, Cambridge, MA, 1986. IEEE Computer Society Press.

[5] J. Despeyroux, A. Felty, and A. Hirschowitz. Higher-order abstract syntax in Coq. In M. Dezani-Ciancaglini and G. Plotkin, editors, Proceedings of the International Conference on Typed Lambda Calculi and Applications, pages 124-138, Edinburgh, Scotland, Apr. 1995. Springer-Verlag LNCS 902.

[6] A. Felty. Two-level meta-reasoning in Coq. In V. A. Carreño, editor, Proceedings of the 15th International Conference on Theorem Proving in Higher Order Logics, volume 2342 of LNCS. Springer Verlag, 2002.

[7] M. Gabbay and A. Pitts. A new approach to abstract syntax involving binders. In G. Longo, editor, Proceedings of the 14th Annual Symposium on Logic in Computer Science (LICS'99), pages 214-224, 1999. IEEE Computer Society Press.

[8] L. Hallnas. Partial inductive definitions. TCS, 87(1):115-147, July 1991.

[9] J. Hannan and D. Miller. From operational semantics to abstract machines. Mathematical Structures in Computer Science, 2(4):415-459, 1992.

[10] J. Hannan and F. Pfenning. Compiler verification in LF. In A. Scedrov, editor, Seventh Annual IEEE Symposium on Logic in Computer Science, pages 407-418, Santa Cruz, California, June 1992.

[11] P. H. Hartel and L. Moreau. Formalizing the safety of Java,the Java Virtual Machine, and Java Card. ACMCS, 33(4):517-558, Dec. 2001.

[12] F. Honsell, M. Miculan, and I. Scagnetto. An axiomatic approach to metareasoning on systems in higher-order abstract syntax. In Proc. ICALP'01, number 2076 in LNCS, pages 963-978. Springer-Verlag, 2001.

[13] D. Lester and S. Mintchev. Towards machine-checked compiler correctness for higher-order pure functional languages. In L. Pacholski and J. Tiuryn, editors, Computer Science Logic, pages 369-381. Springer-Verlag LNCS 933, 1995.

[14] R. McDowell and D. Miller. Reasoning with higher-order abstract syntax in a logical framework. ACM Transactions on Computational Logic, 3(1):80-136, 2002.

[15] D. Miller, G. Nadathur, F. Pfenning, and A. Scedrov. Uniform proofs as a foundation for logic programming. Annals of Pure and Applied Logic, 51:125-157, 1991.

[16] R. Milner and M. Tofte. Co-induction in relational semantics. Theoretical Computer Science, 87:209-220, 1991.

[17] A. Momigliano, S. Ambler, and R. Crole. A Hybrid encoding of Howe's method for establishing congruence of bisimilarity. ENTCS, 70(2), 2002.

[18] F. Pfenning. Computation and Deduction. Cambridge University Press, 2000. In preparation. Draft from April 1997 available electronically.

[19] F. Pfenning and E. Rohwedder. Implementing the meta-theory of deductive systems. In D. Kapur, editor, Proceedings of the 11th International Conference on Automated Deduction, pages 537-551. Springer-Verlag LNAI 607.

[20] F. Pfenning and C. Schürmann. System description: Twelf - a meta-logical framework for deductive systems. In H. Ganzinger, editor, Proceedings of CADE 16, pages 202-206. Springer LNAI 1632.

[21] C. Schürmann. Automating the Meta-Theory of Deductive Systems. PhD thesis, Carnegie-Mellon University, 2000. CMU-CS-00-146. 